\title{
The difference scheme for the two-dimensional convection-diffusion problem for large peclet numbers
}

\author{
Alexander I. Sukhinov, Alexander E. Chistyakov ${ }^{*}$ and Yulia V. Belova \\ Don State Technical University, 344000 Rostov-on-Don, Russia
}

\begin{abstract}
The purpose of this work is the development of a difference scheme for the solution of convection-diffusion problem at high Peclet numbers $(\mathrm{Pe}>2)$. In accordance with this purpose the following problems were solved: difference scheme for convection is built, comparison with the existing schemes is carried out; conditions for stability of the proposed difference scheme are obtained. Solutions of the convection-diffusion equation on the basis of the proposed difference scheme at various Peclet numbers are obtained.
\end{abstract}

\section{Introduction}

The paper deals with the problem of increasing the accuracy of the numerical solution of the convection-diffusion equation for large Peclet numbers. The accuracy of simulation based on finite difference schemes decreases with increasing Peclet number, but the MonteCarlo method, based on the functional integration technique, works the better, when this number larger. The Monte-Carlo method does not have sufficient accuracy, and the addition of nodes does not guarantee neither an increase in the accuracy nor stability of the solution in the class of two-layer difference schemes. The schemes with central differences, which work with a Peclet number less than two, are more precise. Often in practice, in order to use these schemes, it is necessary to increase the dimension of the grids, which entails a considerable increase in the computational effort.

Another approach is the introduction of dissipative terms, which adversely affects the accuracy of the solution. The "left corner" scheme is an example of a dissipative scheme. The approach associated with the use of dissipative schemes for the calculation of transport equations is highly undesirable.

In the class of three-layer difference schemes, there are "CABARET" schemes that possess the property of stability and nondissipativity, but are inferior in accuracy to the central difference scheme in the case of its applicability. Thus, the construction of a difference scheme that is superior in accuracy to the "CABARET" scheme and has the properties of stability and nondissipativity in the case of Peclet numbers greater than two is an actual problem.

\footnotetext{
*Corresponding author: cheese_05@mail.ru
} 


\section{The difference scheme}

Let consider the transfer equation

$$
\frac{\partial q}{\partial t}+u \frac{\partial q}{\partial x}=0
$$

where $t \in[0, T], x \in[0, L], q(0, x)=q^{0}(x), q(t, 0)=q(t, L)=0, u=$ const .

Let introduce the uniform grid $\omega=\bar{\omega}_{h} \times \omega_{\tau}$, where $\bar{\omega}_{h}=\left\{x_{i} \mid x_{i}=i h, i=0,1, \ldots, N, N h=L\right\}, \omega_{\tau}=\left\{t_{j} \mid j=0,1, \ldots\right\}, \tau=t_{n+1}-t_{n}=$ const.

To solve the problem numerically, it is possible to use the following finite-difference schemes [1]

- the "anti-flow" scheme or for $u>0$ the left corner [1]

$$
\frac{q_{i}^{n+1}-q_{i}^{n}}{\tau}+u \frac{q_{i}^{n}-q_{i-1}^{n}}{h}=0
$$

- the central difference scheme

$$
\frac{q_{i}^{n+1}-q_{i}^{n}}{\tau}+u \frac{q_{i+1}^{n}-q_{i-1}^{n}}{2 h}=0 ;
$$

- the "CABARET" scheme [2]

$$
\frac{q_{i}^{n+1}-q_{i}^{n}}{2 \tau}+\frac{q_{i-1}^{n}-q_{i-1}^{n-1}}{2 \tau}+u \frac{q_{i}^{n}-q_{i-1}^{n}}{h}=0 .
$$

The scheme obtained as a result of a linear combination of the central difference scheme (3) and the "CABARET" scheme can be used to solve the convection problem (4)

$$
\begin{gathered}
\frac{1}{2}\left(\frac{q_{i}^{n+1}-q_{i}^{n}}{\tau}+u \frac{q_{i+1}^{n}-q_{i-1}^{n}}{2 h}\right)+\frac{q_{i}^{n+1}-q_{i}^{n}}{2 \tau}+\frac{q_{i-1}^{n}-q_{i-1}^{n-1}}{2 \tau}+u \frac{q_{i}^{n}-q_{i-1}^{n}}{h}=0 ; \\
\frac{q_{i}^{n+1}-q_{i}^{n}}{\tau}+\frac{q_{i-1}^{n}-q_{i-1}^{n-1}}{2 \tau}+u \frac{q_{i+1}^{n}+4 q_{i}^{n}-5 q_{i-1}^{n}}{4 h}=0 .
\end{gathered}
$$

The grid pattern for the proposed scheme is shown in Fig. 1.

$\mathrm{t}$

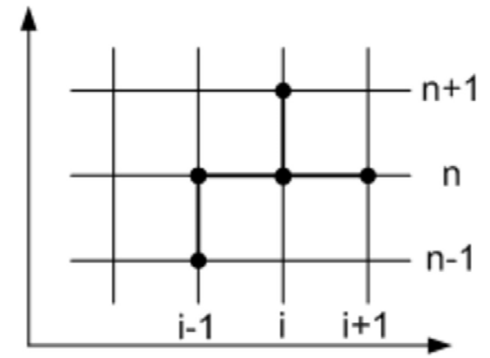

Fig. 1. The grid template on which the new scheme is defined. 
Let find the numerical solutions based on the schemes (2) - (5) of the model problem with initial conditions $q^{0}(x)=h(20-\mathrm{x})$, where $h(\mathrm{x})-$ Heaviside function. The solutions of the model problem (1 - numerical solution, 2 - exact solution) are shown in Fig. 2.
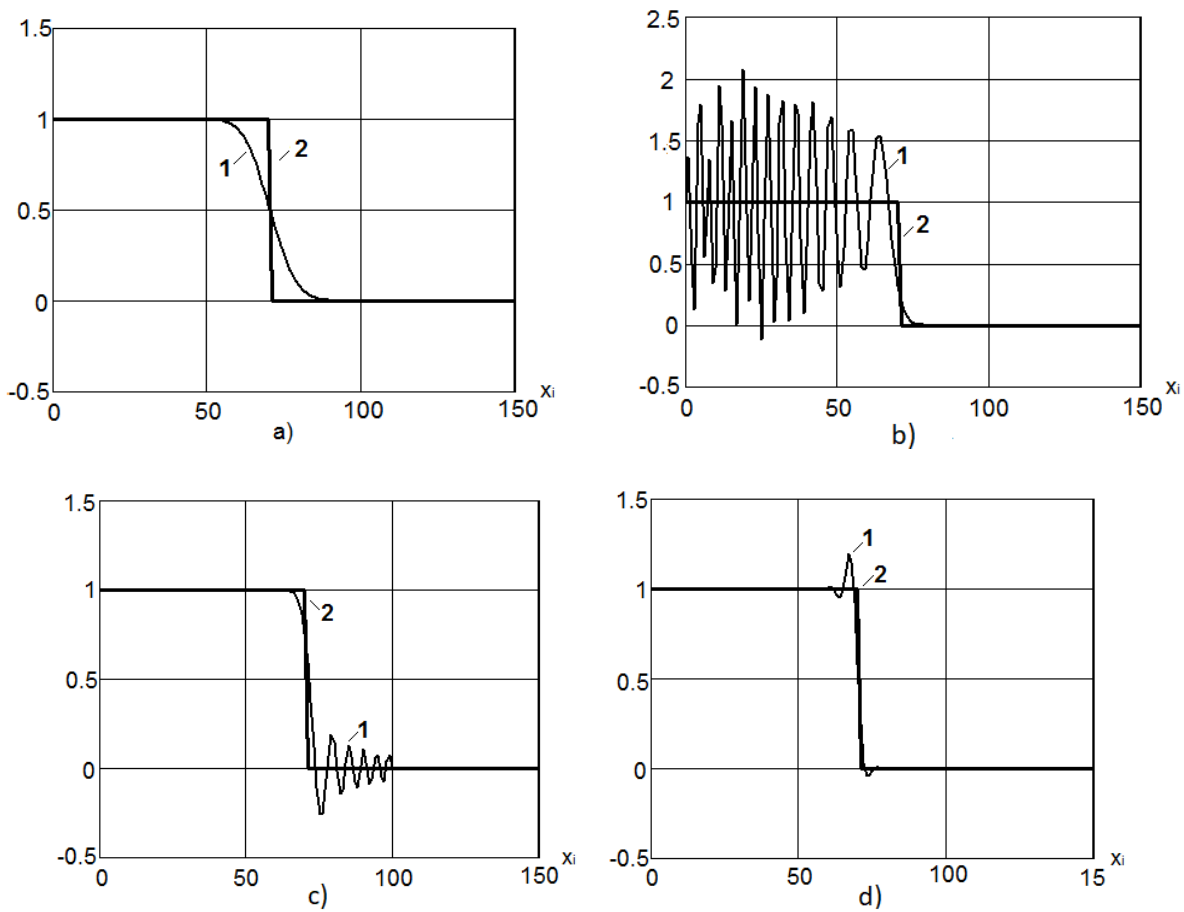

Fig. 2. The solutions of the model problem based on the schemes (2) - (5): a) the left difference scheme; b) the central difference scheme; c) the "CABARET" scheme; d) the proposed difference scheme.

It is seen from the figure that the left difference scheme approximates the "jump" insufficiently accurately, the central difference scheme is unstable, the solution obtained on the basis of the "CABARET" scheme has oscillations (entropy perturbations) [3]. The proposed difference scheme gives the most accurate solution of the model problem. The left difference scheme has the order of approximation $O(\tau+h)$, the central difference scheme approximates the continuous model with the order $O\left(\tau+h^{2}\right)$, scheme "CABARET" - with the order $O\left(\tau^{2}+h^{2}\right)$.

\section{The approximation of the convection-diffusion problem}

Consider the nonstationary convection-diffusion equation

$$
\frac{\partial q}{\partial t}+u \frac{\partial q}{\partial x}=\mu \frac{\partial^{2} q}{\partial x^{2}}
$$

where $t \in[0, T], x \in[0, L], q(0, x)=q^{0}(x), q(t, 0)=q(t, L)=0, u=$ const. 
The scheme obtained as a result of a linear combination of the central difference scheme (3) and the cabaret scheme (4) will be used to approximate the convection operator

$$
\begin{gathered}
\frac{1}{2}\left(\frac{q_{i}^{n+1}-q_{i}^{n}}{\tau}+u \frac{q_{i+1}^{n}-q_{i-1}^{n}}{2 h}-\mu \frac{q_{i+1}^{n}-2 q_{i}^{n}+q_{i-1}^{n}}{h^{2}}\right)+ \\
+\frac{q_{i}^{n+1}-q_{i}^{n}}{2 \tau}+\frac{q_{i-1}^{n}-q_{i-1}^{n-1}}{2 \tau}+u \frac{q_{i}^{n}-q_{i-1}^{n}}{h}-\mu \frac{q_{i+1}^{n}-2 q_{i}^{n}+q_{i-1}^{n}}{h^{2}}=0 ; \\
\frac{q_{i}^{n+1}-q_{i}^{n}}{\tau}+\frac{q_{i-1}^{n}-q_{i-1}^{n-1}}{2 \tau}+u \frac{q_{i+1}^{n}+4 q_{i}^{n}-5 q_{i-1}^{n}}{4 h}-3 \mu \frac{q_{i+1}^{n}-2 q_{i}^{n}+q_{i-1}^{n}}{2 h^{2}}=0 .
\end{gathered}
$$

In Figure 3, the solutions of the model problem with initial conditions $q^{0}(x)=h(20-\mathrm{x})$, where $h(\mathrm{x})$ - Heaviside function $(1-$ the exact solution of the transfer problem, 2 - the numerical solution based on the proposed difference scheme for different values of Peclet numbers, 3 - the numerical solution of the transfer problem based on the "against" flow).
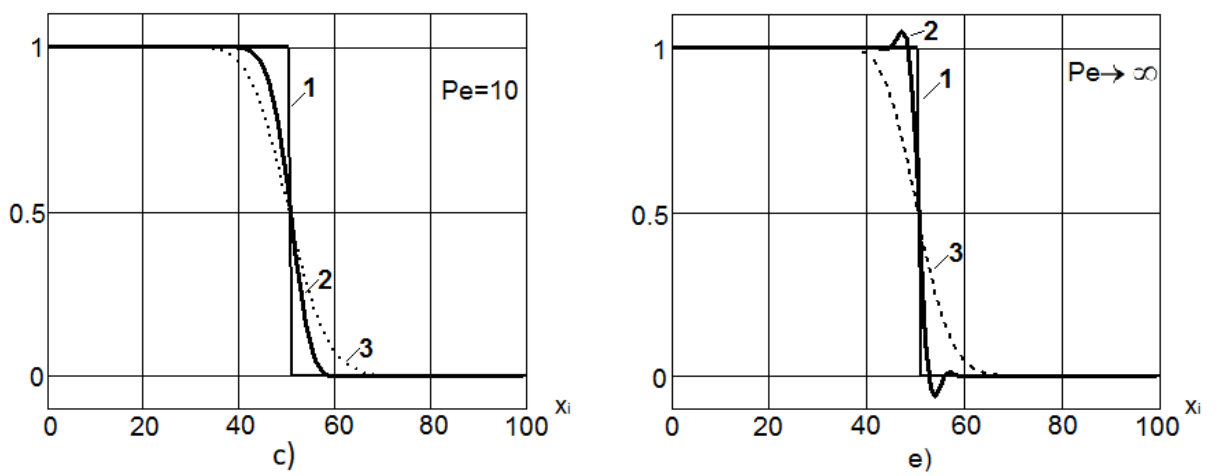

Fig. 3. The solutions of the model problem of concentrations front transport: 1 - the exact solution of the transfer problem, 2 - the numerical solution based on the proposed difference scheme for different values of Peclet numbers, 3 - the numerical solution of the transfer problem based on the "against" flow.

\section{The two-dimensional diffusion-convection problem}

Consider the two-dimensional convection-diffusion equation

$$
\frac{\partial q}{\partial t}+u \frac{\partial q}{\partial x}+v \frac{\partial q}{\partial y}=\mu \frac{\partial^{2} q}{\partial x^{2}}+\mu \frac{\partial^{2} q}{\partial y^{2}},
$$

where $t \in[0, T], x \in\left[0, L_{x}\right], y \in\left[0, L_{y}\right] q(0, x, y)=q^{0}(x, y), q(t, 0, y)=q\left(t, L_{x}, y\right)=0$, $q(t, x, 0)=q\left(t, x, L_{y}\right)=0$. 
Cover the domain of definition by a uniform computational grid $\omega=\omega_{\tau} \times \bar{\omega}_{x} \times \bar{\omega}_{y}$, where $\bar{\omega}_{x}=\left\{x_{i} \mid x_{i}=i h_{x}, i=0,1, \ldots, N, N h_{x}=L_{x}\right\}, \bar{\omega}_{y}=\left\{y_{i} \mid y_{i}=i h_{y}, i=0,1, \ldots, N, N h_{y}=L_{y}\right\}$, $\omega_{\tau}=\left\{t_{j} \mid j=0,1, \ldots\right\}, \tau=t_{n+1}-t_{n}=$ const.

The simulation was performed on a grid with the dimensions $100 \times 100$ computed nodes with the follow parameters: the dimensions of the computed area $L_{x}=100 \mathrm{~m}, L_{y}=100 \mathrm{~m}$, the horizontal component is $4 \mathrm{~m} / \mathrm{c}$, vertical $-3 \mathrm{~m} / \mathrm{c}$, the turbulent exchange coefficient was set equal to: $0,0.1,0.4,2 \mathrm{~m}^{2} / \mathrm{c}$. In solving the two-dimensional convection-diffusion problem, the initial distribution was given by the function [4]:

$$
q(x, y)=\left\{\begin{array}{l}
\sin (\pi(x-10) / 10) \sin (\pi(y-10) / 10),\{x, y\} \in D, \\
0,\{x, y\} \notin D, \\
D:\{x \in[10,20], y \in[10,20]\}
\end{array}\right.
$$

The numerical solution of the convection problem with help of the difference scheme (7) at different time intervals is shown in Fig. 4.
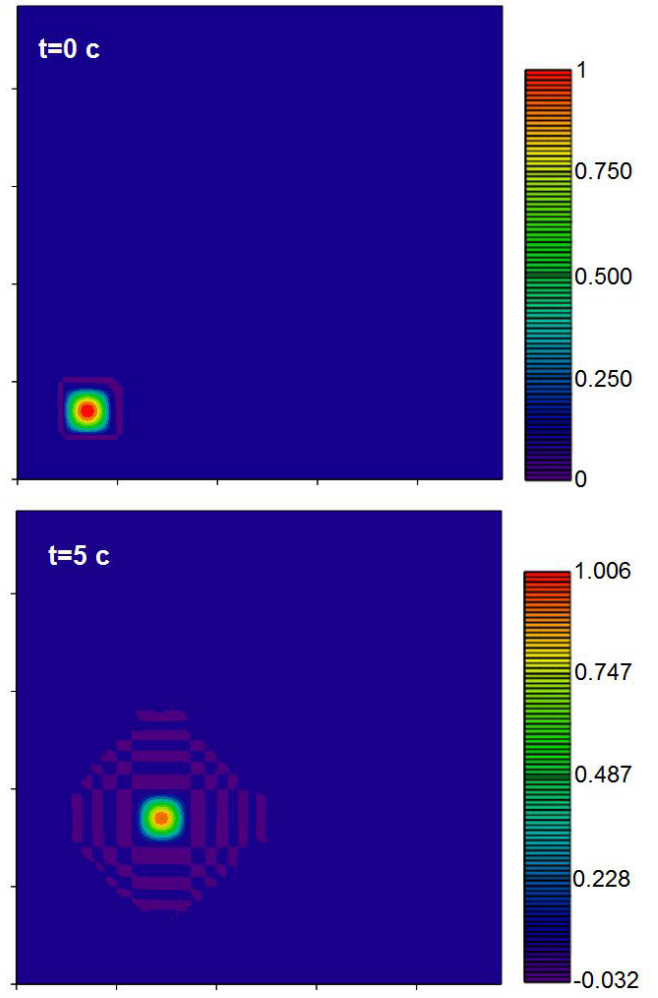
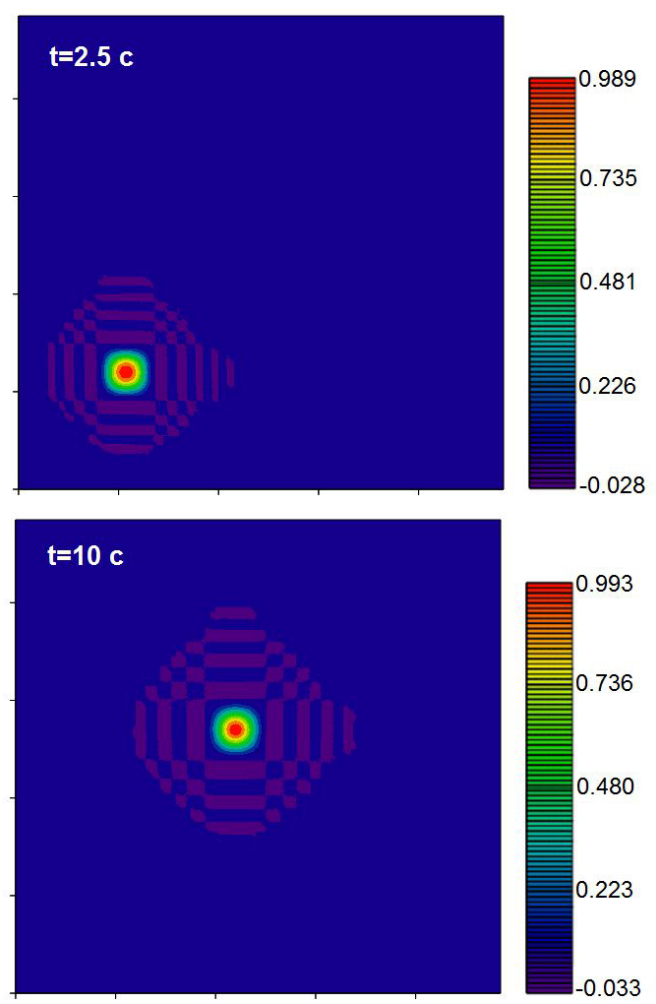

Fig. 4. The numerical solution of the convection problem using the scheme (5) at different time intervals. 


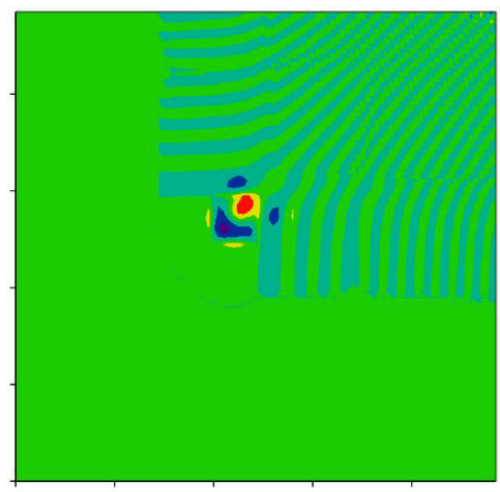

a)

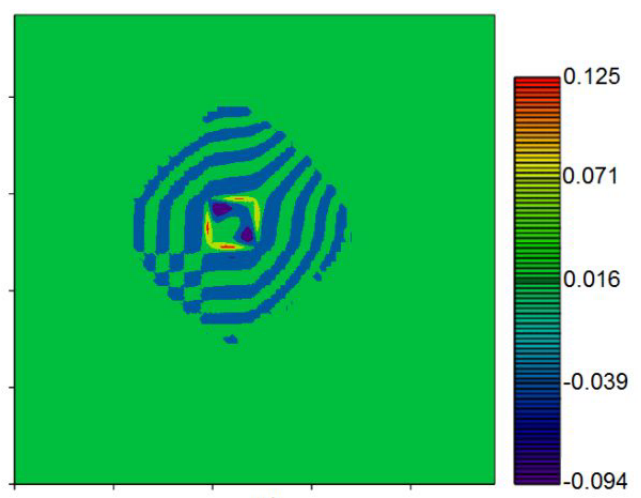

b)

Fig. 5. The error in the numerical solution of the convection problem $(\mu=0, P e \rightarrow \infty)$ using a) the "CABARET" scheme and b) the scheme (5).

The limiting absolute error in solving the convection problem with help of the "CABARET" scheme is 0.281 units, and using the scheme (5), 0.125 units. Thus, when $P e \rightarrow \infty$ the error in the numerical solution of the model problem (9) with help of the difference scheme (5) is 2.248 times less than the error in solving with help of the "CABARET" scheme. Figure 5 shows the errors of the numerical solution of the convection problem for various schemes.

\section{Conclusion}

When modeling applied problems, such as the problem of hydrodynamics of shallow water bodies, aerodynamics, population dynamics and others, it is necessary to solve the convection-diffusion equations. If the problem poses the condition for the predominance of convection over diffusion, then the standard schemes have a low accuracy. The paper proposes a three-layer difference scheme for the convection-diffusion equation, which is a linear combination of the "CABARET" scheme and the central difference scheme. It is shown that the new scheme is stable for Courant numbers belonging to the interval $[0,1]$ and large Peclet numbers $(P e>20)$. An investigation of the numerical solution of the two-dimensional convection-diffusion equation in the limiting case (the diffusion coefficient is zero) shows that for large Peclet numbers the error in the numerical solution of the model problem based on the proposed difference scheme is 2.248 times smaller than the error in solving with the "CABARET" scheme.

This paper was partially supported by the grant No.17-11-01286 of the Russian Science Foundation.

\section{References}

1. A.I. Sukhinov, Yu.V. Belova, A.E. Chistyakov, Vychislitelnye metody i programmirovanie, 18, 4, 371 (2015).

2. V.A. Gushchin, Mathematical Models and Computer Simulations, 8, 5, 487 (2016)

3. O.M. Belotserkovskii, V.A. Gushchin, V.N. Kon'shin, USSR Computational Mathematics and Mathematical Physics, 27, 2, 181 (1987)

4. A.A. Samarskiy, P.N. Vabishchevich, Numerical methods for solving convectiondiffusion problems (Editorial URSS, Moscow, 1999) 
5. V.M. Goloviznin, A.A. Samarskii, Mathematical Models and Computer Simulations, 10, 1, 86 (1998)

6. M.E. Ladonkina, O.A. Neklyudova, V.F. Tishkin, Mathematical Models and Computer Simulations, 6, 4, 397 (2014)

7. A.A. Samarskii, U.S.S.R. Comput. Math. Math. Phys., 7, 1, 62 (1967)

8. A.A. Samarskii, U.S.S.R. Comput. Math. Math. Phys., 7, 5, 171 (1967)

9. R.P. Fedorenko, U.S.S.R. Comput. Math. Math. Phys., 2, 6, 1355 (1963)

10. A.I. Sukhinov, A.E. Chistakov, M.V Iakobovskii, Vestn. YuUrGU. Ser. Vych. Matem. Inform., 5, 1, 47 (2016)

11. A.I. Sukhinov, D.S. Khachunts, A.E., Computational Mathematics and Mathematical Physics, 55, 7, 1216 (2015)

12. A.I. Sukhinov, A.E. Chistyakov, E.V. Alekseenko, Mathematical Models and Computer Simulations, 3, 5, 562 (2011)

13. A.I. Sukhinov, A.E.Chistyakov, E.A. Protsenko, Mathematical Models and Computer Simulations, 6, 4, 351 (2014)

14. A.I. Sukhinov, A.E. Chistyakov, E.F. Timofeeva, A.V.Shishenya, Mathematical Models and Computer Simulations, 5, 2, 122 (2013)

15. A.I. Sukhinov, A.E. Chistyakov, Mathematical Models and Computer Simulations, 4, 4, 398 (2012)

16. A.I. Sukhinov, A.E. Chistyakov, I.I. Levin, I.S. Semenov, A.V. Nikitina, A.A. Semenyakina, 2016 5th International Conference on Informatics, Electronics and Vision, ICIEV 2016, 1128 (2016)

17. A. Sukhinov, A. Chistyakov, A. Nikitina, A. Semenyakina, I. Korovin, G. Schaefer, 2016 5th International Conference on Informatics, Electronics and Vision, ICIEV 2016, 1134 (2016)

18. A.V. Nikitina, A.I. Sukhinov, G.A. Ugolnitsky, A.B. Usov, A.E. Chistyakov, M.V. Puchkin, I.S. Semenov, Mathematical Models and Computer Simulations, 9, 1, 101 (2017)

19. A.I. Sukhinov, A.E. Chistyakov, A.V. Shishenya, Mathematical Models and Computer Simulations, 6, 3, 324 (2014)

20. N. Buzalo, P. Ermachenko, T. Bock, A. Bulgakov, A. Chistyakov, A. Sukhinov, E. Zhmenya, (...), N. Zakharchenko, Procedia Engineering, 85, 84 (2014) 